\title{
REVIEW
}

\section{A Review of Tafamidis for the Treatment of Transthyretin-Related Amyloidosis}

\author{
Márcia Waddington Cruz · Merril D. Benson
}

To view enhanced content go to www.neurologytherapy-open.com

Received: June 1, 2015 / Published online: August 15, 2015

(C) The Author(s) 2015. This article is published with open access at Springerlink.com

\section{ABSTRACT}

Transthyretin (TTR)-related amyloidosis (ATTR) is a devastating disease which affects a combination of organs including the heart and the peripheral nerves, and which has a fatal outcome if not treated within a average of 10 years. Tafamidis, or 2-(3,5-dichloro-phenyl)benzoxazole-6-carboxylic acid, selectively binds to TTR with negative cooperativity and kinetically stabilizes wild-type native TTR and mutant TTR; tafamidis therefore has the potential to halt the amyloidogenic cascade initiated by TTR tetramer dissociation, monomer misfolding, and aggregation. The first tafamidis trial, Fx-005, evaluated the effect of 18 months of tafamidis treatment

Electronic supplementary material The online version of this article (doi:10.1007/s40120-015-0031-3) contains supplementary material, which is available to authorized users.

M. Waddington Cruz ( $\square)$

Amyloidosis Research and Treatment Center (CEPARM), University Hospital (HUCFF), Federal University of Rio de Janeiro, Rio De Janeiro, Brazil e-mail: mwaddingtoncruz@gmail.com

\section{D. Benson}

Department of Pathology and Laboratory Medicine, Indiana University School of Medicine,

Indianapolis, IN, USA
(20 mg once daily) on disease progression, as well as assessing its safety in TTR-FAP Val30Met patients. The secondary objective of this trial was to study the pharmacodynamic stabilization of mutated TTR. Tafamidis proved effective in reducing the progress of neuropathy, and in maintaining the nutritional status and quality of life of stage 1 (able to walk without support) Val3OMet TTRFAP patients. Furthermore, TTR stabilization was achieved in more than $90 \%$ of patients. An extension study, Fx-006, was conducted to determine the long-term safety and tolerability of tafamidis and to assess the efficacy of the drug on slowing disease progression. No significant safety or tolerability issues were noticed. Taken together, the results from both trials indicated that the beneficial effects of tafamidis were sustained over a 30-month period and that starting treatment early is desirable. Results are expected from an extended open-label study but data that have already been presented show that long-term use of tafamidis in Val30Met patients is associated with reduced progression in polyneuropathy. Tafamidis was initially approved for commercial use in Europe in 2011 and has since been 
approved for use in Japan, Mexico, and Argentina where it is used as a first-line treatment option for patients with early-stage TTR-FAP. Patients should be carefully followed at referral centers to ascertain the individual response to treatment. In cases of discontinuation, liver transplantation and enrollment in clinical trials of novel drugs aimed mostly toward suppression of TTR production are options.

Keywords: Amyloidosis; Familial amyloid polyneuropathy; Tafamidis; Transthyretin

\section{INTRODUCTION}

Transthyretin familial amyloid polyneuropathy (TTR-FAP) is a devastating disease which not only robs patients of their quality of life (QOL)-mainly from nerve and heart dysfunction-but also promises to inflict a similar fate on their children [1, 2]. An effective treatment for TTR-FAP is sorely needed. There are currently two therapeutic approaches which have proven effective in the treatment of TTR-FAP caused by either abnormal or dysfunctional proteins: One is to interrupt the pathways through which TTR causes TTR-FAP's pathology; the other is to eliminate (or at least decrease) the production of the protein which leads to amyloid fibril formation.

The first therapeutic approach to be tested for TTR-FAP was liver transplantation (LT). This eliminated the production of the causative TTR mutant by the liver [3]. Although this therapy has met with success, it is often limited as a result of continued amyloid fibril formation from wild-type (WT) TTR which results in disease progression in some patients, even though $98 \%$ of mutated TTR is no more produced. The need for other therapeutic approaches was therefore apparent.

The idea of altering the pathway of TTR to amyloid fibril formation was conceived from the in vitro observation that mutations in the TTR protein destabilized its tetrameric structure and enhanced amyloid fibril formation [4, 5]. If this destabilization was a factor in amyloid pathogenesis, therapeutic agents which stabilized the structure of TTR might therefore interfere with amyloid formation. Further studies revealed that the binding of thyroxine to TTR resulted in the stabilization of TTR in solution $[4,5]$. This led to a search for nontoxic, small organic compounds which could bind to the TTR-thyroxine-binding pocket and, hence, stabilize TTR's structure. Diflunisal, an anti-inflammatory drug marketed for treatment of arthritis, was shown to do this [6], and this led to further studies to find safe synthetic compounds that would selectively stabilize TTR and that had greater TTR-stabilizing potency.

\section{THE DISCOVERY OF TAFAMIDIS AS A POTENTIAL TREATMENT FOR TTR-FAP}

As mentioned above, mutations in TTR destabilize its homotetrameric structure, and lead to its dissociation into monomers that misfold and misassemble into tissues as amyloid deposits [4, 5]. Amyloidogenesis is certainly responsible for neurodegeneration in TTR-FAP [7-10, 41].

An in vitro analysis of material from patients of Portuguese descent who inherited one copy of the Val30Met TTR gene, with the presence of a second mutation-namely $\mathrm{T} 119 \mathrm{M}$-on their second allele, showed that this resulted in the formation of T119 M/Val30Met TTR heterotetramers $[4,5,11,12]$. These are more 
stable than the heterotetramers which comprise the Val30Met and WT subunits, and the more stable they are the less likely it is that they will dissociate into the monomers that aggregate as amyloid fibrils and cause toxicity to tissues [5]. This mechanism is known to ameliorate the phenotypical expression of TTR-FAP [4, 5]. Based on this concept, a compound that could selectively bind to the TTR tetrameric structure and increase the kinetic barrier for tetramer dissociation similar to the heterozygous compound T119 M/Val30Met TTR was searched for [4].

Of the multiple TTR kinetic stabilizers reported, benzoxazoles were pursued to identify an oral candidate exhibiting potent and selective TTR binding in blood, while lacking the nonsteroidal anti-inflammatory activity that would be deleterious to patients with cardiomyopathy (CM). Tafamidis, or 2(3,5-dichloro-phenyl)-benzoxazole-6-carboxylic acid, was the compound chosen for clinical development. Tafamidis selectively binds TTR with negative cooperativity and kinetically stabilizes WT TTR and mutant TTR under fibrilpromoting conditions (at $\mathrm{pH} 4.5$ ), denaturing conditions (high urea concentration), and physiological conditions, and therefore has the potential to halt the amyloidogenic cascade initiated by tetramer dissociation, monomer misfolding, and aggregation [4]. A study by Bulawa et al. [5] showed that tafamidis was able to stabilize the two most significant mutations, Val30Met and V122I TTR, in the same way as it stabilized WT TTR. In addition, 30 other amyloidogenic variants of TTR were stabilized by tafamidis. Tafamidis dose-dependently decreases the rate of tetramer dissociation at physiological $\mathrm{pH}[4,5]$. Tafamidis binds with high affinity to TTR at its T4-binding sites, and there is no concern of thyroid metabolic effects since less than $1 \%$ of TTR in circulating plasma carries thyroxine $[4,5]$. The primary carrier of thyroxine is thyroxine-binding globulin. Of note, tafamidis also binds selectively to TTR in human plasma $[4,5]$.

Amyloid non-fibril intermediates can also cause toxicity [4]. Therefore, for optimal treatment, it is desired that the amyloidogenic cascade is halted at the beginning-that is, the rate-limiting step of the cascade that is considered to be the dissociation of tetramers into monomers (Fig. 1). For the development of clinical trials on tafamidis, it was hypothesized that TTR stabilization would lead to reduced amyloid deposition and hence less neurodegeneration (Fig. 2) [5].

\section{CLINICAL TRIALS WITH TAFAMIDIS}

\section{FX-005}

The primary objective of the first tafamidis trial, Fx-005, was to evaluate the effect of 18 months of tafamidis treatment ( $20 \mathrm{mg}$ once daily) on disease progression, and to assess its safety in TTR-FAP Val30Met patients [13]. The secondary objective was to study the pharmacodynamic stabilization of mutated TTR. This was a multicenter, international, randomized, placebo-controlled, double-blind trial. Patients with stage 1 TTR-FAP (able to walk without support) with autonomic or sensory-motor neuropathies were enrolled at eight sites from countries in Europe and South America (Table 1). Patients from both sexes, aged 18-75 years, with confirmed amyloid-positive biopsies, Karnofsky functional status $\geq 50$, with no other cause of neuropathy, no major liver or kidney dysfunction, no prior LT, and New York Heart Association (NYHA) status $\leq 3$ were enrolled. The following outcome measures (validated for diabetic neuropathy were used in the absence of any validated measure for 


\section{Functional forms of TTR}

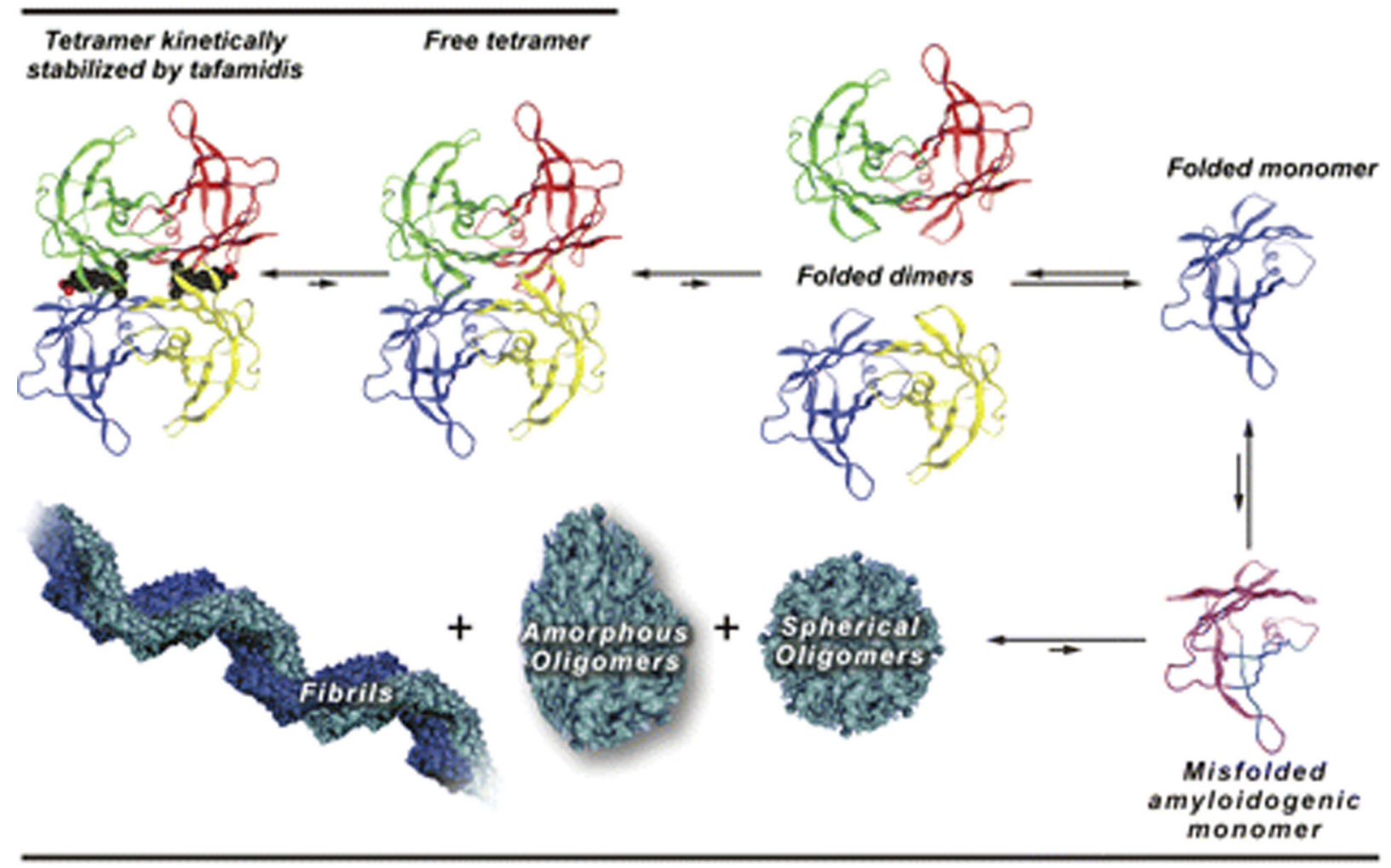

TTR structures associated with pathology

Fig. 1 The amyloidogenic cascade is initiated by tetramer dissociation into monomers that misfold and aggregate causing toxicity. The site of binding of tafamidis is marked. Transthyretin (TTR). Source: with permission from Ref. [5]

TTR-FAP) were used: the neuropathy impairment score in the lower limbs (NIS-LL), which measures motor, sensory, and reflex functions; the Norfolk quality of life-diabetic neuropathy questionnaire (Norfolk QOL-DN), which evaluates several aspects related to sensory and motor neuropathy, as well as autonomic dysfunction reflex on daily activities; modified body mass index (mBMI), which measures autonomic and gastrointestinal function wasting; and summated scores from multiple measures of nerve conduction, quantitative sensory test, and heart rate response to deep breath (HRDB). The summated 7 nerve tests ( $\sum 7$ NTs) assess the large-fiber function of nerves and summated 3 nerve tests ( $\sum 3$ NTs) assess the small-fiber function of peripheral nerves $[14,15]$. TTR stabilization immunoturbidimetric assays were performed on the plasma of patients [13].

The primary efficacy endpoints analyzed in the intent-to-treat (ITT; all randomized patients) population were the NIS-LL response to treatment and least-square (LS) mean change from baseline in Norfolk QOL-DN at 18 months. Responders were considered as those who had an increase in NIS-LL from baseline of $<2$ points. Patients who discontinued due to LT were defined as nonresponders [13].

A further analysis was performed in the population that completed the study. The efficacy-evaluable (EE) population was 
(a)

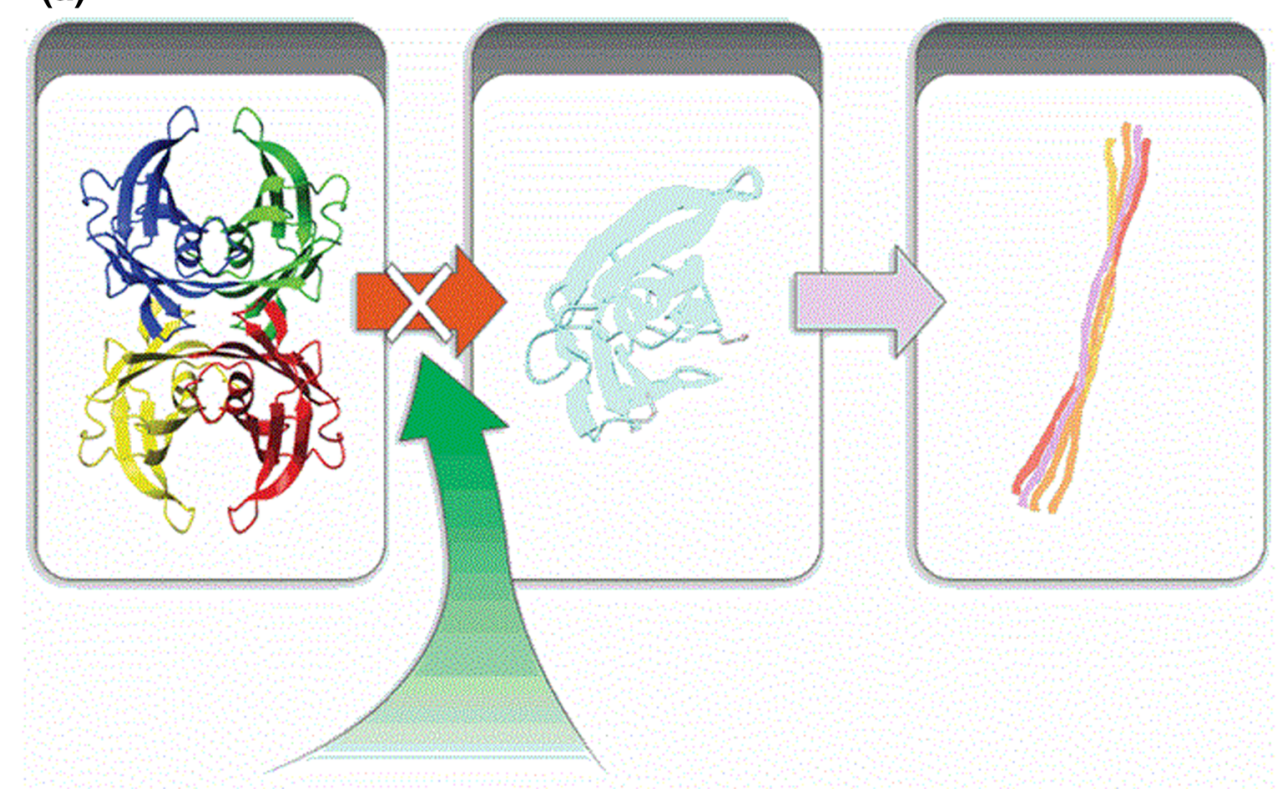

(b)

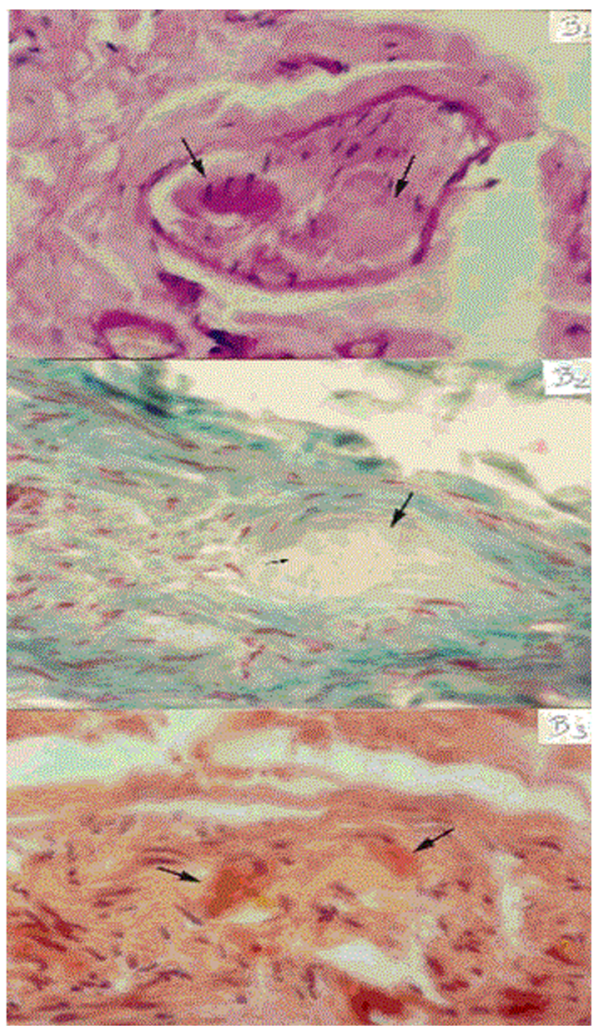

Fig. 2 Mechanism of action of tafamidis binding to thyroxin-binding sites at the transthyretin tetramer preventing dissociation into misfolded monomers and formation of fibrils and amyloid. a Tafamidis prevents dissociation into misfolded monomers and formation of amyloid fibrils. b Amyloid deposits in peripheral nerves in different tissue stainings (H\&E, Gomori trichrome and Congo red) 
prespecified assuming a dropout of patients for LT, as many of them were on its waiting list. As secondary endpoints, change from baseline at months 6, 12, and 18 in NIS-LL, Norfolk QOL-DN, $\sum 7$ NTs, $\sum 3$ NTs, and mBMI were used. Overall, 128 patients were randomized, with $69 \%$ on the waiting list for LT. From this population, $21 \%$ in each treatment arm (active drug or placebo) discontinued to undergo LT. Baseline characteristics of the patients receiving either tafamidis or placebo were similar. Patients were in the early stage of the disease with greater involvement of small-fiber function, in relatively good nutritional status, and had some impairment of QOL [13].

In the ITT population, there was a trend toward more NIS-LL responders in the tafamidis group when compared with the placebo group at month 18 (45.3\% vs. $29.5 \%$, respectively). The change from baseline in Norfolk QOL-DN score in the two groups was not significant at month 18. In the EE population, significantly more patients treated with tafamidis were NIS-LL responders when compared to the placebo-treated group $(60 \%$ vs. $38.1 \%$, respectively, $P=0.041$ ) and a significantly lower LS mean change from baseline in Norfolk QOL-DN in the tafamidis group when compared to the placebo group was observed (0.1 vs. 8.9 points, respectively, $P=0.045$ ) [13].

In the analyses of the secondary endpoints in the ITT population at 6,12 , and 18 months, patients receiving tafamidis showed $52 \%$ less neurologic deterioration in NIS-LL when compared to placebo-treated patients. This difference was due to significantly more weakness in distal lower limbs of the placebo group. Nerve function measured by summated scores were also analyzed in a 6-month interval and showed 5 times greater mean deterioration in small-fiber function ( $\sum 3$ NTs normal deviates [nds]; $P=0.05$ ) for the placebo group and no trend toward more deterioration of large-fiber function ( $\sum 7$ NTs nds; $\left.P=0.066\right)$. mBMI significantly increased from baseline at 18 months (LS mean +39.3) compared to a worsening in patients under placebo $(-33.8)$. Norfolk QOL-DN showed no trend toward preservation of QOL in the tafamidis group $(P=0.209)$. TTR stabilization was demonstrated in $98 \%$ of patients treated with tafamidis. Adverse events were equally distributed in both treatment groups and were mostly related to the disease itself [13].

The Fx-005 trial was a substantial challenge due to the rarity of the disease, its systemic nature, the absence of studies on its natural course, and the fact that there were no devoted validated outcome measures for TTR-FAP. There was also a higher than anticipated dropout rate due to LT (21\% observed vs. 10\% estimated). The choice to undergo LT was equally distributed in both treatment arms and probably reflects the fact that patients had been on the waiting list for LT for a long time and could therefore not afford to wait until the end of the study to make their decision when they were called for LT. Furthermore, LT was considered the standard of care at that time the study was conducted and so patients had a difficult decision on whether to continue using an experimental drug or to undergo LT.

The choice to consider patients who underwent LT as nonresponders possibly influenced the analyses of NIS-LL in the ITT population, probably underpowering the effect on NIS-LL progression. In spite of the inability to demonstrate statistical significance in the primary outcomes, overall the results demonstrated the potential of tafamidis to slow neurologic deterioration and maintain nutritional status. Tafamidis was well tolerated which is important for a continuous-use drug [13]. Future studies would be necessary to study 
Table 1 Summary of tafamidis trials

\begin{tabular}{|c|c|c|c|c|c|}
\hline Protocol & Design & $\begin{array}{l}\text { Number of } \\
\text { participants }\end{array}$ & $\begin{array}{l}\text { Primary } \\
\text { endpoints }\end{array}$ & AEs & Outcomes \\
\hline $\begin{array}{c}\text { Fx-005 } \\
{[13]}\end{array}$ & $\begin{array}{l}\text { Phase II/III, } \\
\text { randomized- } \\
\text { controlled, } \\
\text { double-blind trial } \\
\text { Treatment: placebo } \\
\text { or tafamidis } 20 \mathrm{mg} \\
\text { once daily for } \\
18 \text { months }\end{array}$ & $\begin{array}{l}128 \text { Val30Met } \\
\text { TTR } \\
\text { mutation } \\
\text { proved } \\
\text { patients }\end{array}$ & $\begin{array}{l}\text { NIS-LL, } \\
\text { Norfolk } \\
\text { QOL-DN }\end{array}$ & $\begin{array}{l}\text { Same incidence of AEs and } \\
\text { SAEs in both treatment } \\
\text { arms; urinary infection and } \\
\text { diarrhea more frequent in } \\
\text { the placebo group }\end{array}$ & $\begin{array}{l}\text { EE population NIS-LL } \\
\text { responders: } 60 \% \text { in tafamidis } \\
\text { group, } 38.1 \% \text { in placebo group } \\
(P=0.041) \text {; EE TQoL change } \\
\text { from baseline: } 0.1 \text { in tafamidis } \\
\text { group, } 8.9 \text { in placebo group } \\
(P=0.045)\end{array}$ \\
\hline $\begin{array}{c}\text { Fx-006 } \\
{[16]}\end{array}$ & $\begin{array}{l}\text { Single-arm, open- } \\
\text { label extension of } \\
\text { Fx-005 to evaluate } \\
\text { safety and efficacy } \\
\text { of tafamidis } \\
\text { Treatment: tafamidis } \\
20 \text { mg once daily } \\
\text { for } 12 \text { months }\end{array}$ & $\begin{array}{l}86 \text { Val30Met } \\
\text { TTR } \\
\text { mutation } \\
\text { proved } \\
\text { patients }\end{array}$ & $\begin{array}{l}\text { NIS-LL, } \\
\text { Norfolk } \\
\text { QOL-DN }\end{array}$ & No new safety signs & $\begin{array}{l}\text { Beneficial effects of tafamidis } \\
\text { were sustained over a } \\
\text { 30-month period with gradual } \\
\text { NIS-LL increase to month } 30 \text {; } \\
\text { early-start treatment is } \\
\text { desirable }\end{array}$ \\
\hline $\begin{array}{l}\text { FX1A- } \\
201 \\
{[17]}\end{array}$ & $\begin{array}{l}\text { Multicenter, open- } \\
\text { label trial } \\
\text { Treatment: tafamidis } \\
20 \text { mg once daily } \\
\text { for } 12 \text { months }\end{array}$ & $\begin{array}{l}21 \text { TTR } \\
\text { mutation } \\
\text { patients } \\
\text { excluding } \\
\text { Val30Met } \\
\text { and } \\
\text { Val122Ile }\end{array}$ & $\begin{array}{l}\text { TTR } \\
\text { stabilization } \\
\text { at week } 6\end{array}$ & $\begin{array}{l}\text { Most common AEs: falls } \\
(24 \%) \text {, diarrhea }(24 \%) \text {, and } \\
\text { extremity pain }(19 \%)\end{array}$ & $\begin{array}{l}\text { TTR stabilization at week } 6 \text { was } \\
\text { achieved in } 18(94.7 \%) \text { of } 19 \\
\text { patients in the ITT population }\end{array}$ \\
\hline $\begin{array}{l}\text { FX1B- } \\
201 \\
{[21]}\end{array}$ & $\begin{array}{l}\text { Phase II, open-label } \\
\text { trial } \\
\text { Treatment: tafamidis } \\
20 \mathrm{mg} \text { once daily } \\
\text { for } 12 \text { months } \\
\text { with routine } \\
\text { standard of care }\end{array}$ & $\begin{array}{l}31 \text { wild-type } \\
\text { CM patients } \\
\text { and } 4 \text { TTR } \\
\text { V122I CM } \\
\text { patients }\end{array}$ & $\begin{array}{l}\text { TTR } \\
\text { stabilization } \\
\text { at week } 6\end{array}$ & $\begin{array}{l}\text { Well tolerated; } \\
7 \text { of } 31 \text { wild-type CM patients } \\
\text { had bouts of diarrhea }\end{array}$ & $\begin{array}{l}96.8 \% \text { achieved TTR } \\
\text { stabilization after } 6 \text { weeks; } \\
\text { Kaplan-Meier analysis of } \\
\text { survival from time of diagnosis } \\
\text { was } 87.5 \% \text { at } 30 \text { months; } \\
25.7 \% \text { experienced at least one } \\
\text { cardiovascular-related } \\
\text { hospitalization; } 25.7 \% \\
\text { experienced the composite } \\
\text { endpoint of death or } \\
\text { cardiovascular-related } \\
\text { hospitalization; NYHA was } \\
\text { improved or preserved for } 75 \% \\
\text { of patients after } 12 \text { months }\end{array}$ \\
\hline
\end{tabular}

AEs adverse events, $C M$ cardiomyopathy, EE efficacy evaluable, NIS-LL neuropathy impairment score in the lower limbs, Norfolk $Q O L-D N$ Norfolk quality of life-diabetic neuropathy questionnaire, $N Y H A$ New York heart association, $S A E s$ serious adverse events, $T T R$ transthyretin, TQoL quality of life

the long-term effects of tafamidis on disease progression.

\section{Fx-006}

The extension study, Fx-006, was conducted to determine the long-term safety and tolerability of tafamidis, and to assess its efficacy on slowing disease progression [16]. Patients with TTR-FAP who completed the previous study [13] were eligible to enter this open-label, multicenter, international, single-arm trial in which all patients received tafamidis (20 mg once daily) for 12 months. Patients who were randomized 
to the placebo arm in the previous study were switched to the active drug arm and constituted the placebo-tafamidis group; patients who were on tafamidis in the previous study were continued on tafamidis and constituted the tafamidis-tafamidis group. Values obtained at the 18-month visit of the previous study were considered as baseline values for the extension study, except for 14 patients who went off-drug for a period for more than 2 months due to delays in regulatory approval at their sites. For these patients, new baseline values were obtained and they were not included in the ITT population [16].

Efficacy measures were the same as for the previous study [13-15] and were conducted every 6 months on the ITT population [16]. Adverse events were monitored for the duration of the study. Additional safety measures included 12-lead electrocardiogram (EKG), Holter monitoring, laboratory tests, vital signs, physical examination, ophthalmologic evaluation with fundal photography, and echocardiogram. TTR stabilization was continuously measured by an immunoturbidimetric validated assay $[13,16]$.

To assess efficacy, three hypotheses were proposed. First, that the effect of tafamidis to slow disease progression could be sustained for additional 12 months. To analyze this, the monthly rate of change during the first 18 months was compared to the monthly rate of change during the extension period of the study for the tafamidis-tafamidis population. Second, that tafamidis given to patients who were on placebo in Fx-005 [13] could slow disease progression. To evaluate this, the monthly rate of change of several outcomes was compared during Fx-006 and Fx-005 in the placebo-tafamidis group. Finally, that earlier treatment with tafamidis resulted in better outcomes. Changes in several efficacy measures from the baseline of Fx-005 [13] with month 12 of Fx-006 were compared in the tafamidis-tafamidis group and in the placebotafamidis group. Muscle weakness at the individual joint locations was also evaluated for early-start treatment effect [16].

From the 91 patients who completed Fx-005 study [13], 86 (94.5\%) were enrolled in Fx-006 [16]. Five patients decided not to participate in the extension phase. Of the 86 patients that were enrolled, 71 were considered as the ITT population since 14 (16.3\%) were excluded due to an interruption in treatment of more than 2 months between the studies and one other who did not receive tafamidis. Sixty-three $(88.7 \%)$ completed the extension study. The demographic characteristics of both groups were similar, although patients from the placebo-tafamidis arm presented greater disease severity as could be noticed by their NIS-LL values and other parameters at the start of the Fx-006 study [16].

In the tafamidis-tafamidis group, there were no statistically significant differences in the monthly rate of change in measures including NIS-LL, Norfolk QOL-DN, $\sum 7$ NTs, and $\sum 3$ NTs scores when comparing the first 18 months of the previous study with the 12 months of the extension study [16]. The monthly rate of change of mBMI dropped in the tafamidistafamidis group after entry into Fx-006, but remained higher than those observed prior to treatment. By comparing the rate of progression of several outcomes in the placebo-tafamidis group between the two phases of the study, a significant reduction in the rate of neurologic and neurophysiologic deterioration was observed (NIS-LL monthly rate of change, Fx-005: 0.34; Fx-006: 0.16; $P=0.01)$. The deterioration attested by the Norfolk QOL-DN score during Fx-005 was arrested in Fx-006 in this group (monthly rate of change: -0.16 ; 
$P<0.01)$ and there was a positive rate of change of $\mathrm{mBMI}$ as opposed to a decline observed in Fx-005 $(-1.77 ; P<0.0001)$ [16].

To test the third hypothesis, the monthly rate of change between the two patient populations was compared over the period of 30 months in all efficacy measures. There were significant treatment group (tafamidis-tafamidis vs. placebo-tafamidis) differences in the mean change from baseline $\mathrm{Fx}-005$ at 30 months for NIS-LL (3.0 points vs. 6.8 points; $P=0.04$ ) and for $\sum 7$ NTs nds (1.6 vs. $4.4 ; P<0.01$ ) [16]. No statistically significant difference in the monthly rate of change for Norfolk QOL-DN and $\mathrm{mBMI}$ was noted at 30 months. The mBMI reversed to a positive value for the placebo-tafamidis group in the extension study and might have accounted for this lack of difference. TTR stabilization was achieved in both treatment groups and was more than $90 \%$. No new safety or tolerability issues were noticed. Taken together, the results indicated that the beneficial effects of tafamidis were sustained over a 30-month period and that early-start treatment is desirable [16]. Long-term data are expected from another open-label extension study (see Fx1A-303, below).

\section{Fx1A-201}

In addition to the Fx-005 study, a parallel study was conducted in patients with TTR-FAP due to non-Val3OMet mutations to assess the effect of tafamidis on TTR stabilization and clinical outcomes in these variants [17]. Fx1A-201 was a 12-month study that enrolled patients from centers in Europe and the USA; the study had an open-label design and was divided into two parts. In the first part, patients who received a daily dose of $20 \mathrm{mg}$ of tafamidis were studied at week 6 for TTR stabilization. In the second part, those who demonstrated TTR stabilization were followed for a total of 12 months. The main exclusion criteria was the presence of Val3OMet or Val122Ile TTR mutations. TTR stabilization was studied using the same method as in the previous studies $[13,16]$ and was considered a primary outcome at week 6 .

As secondary endpoints, TTR stabilization at months 6 and 12 and the neurologic impairment score (NIS)—which captures clinically meaningful changes in neurological function (e.g., sensation, muscle weakness, and reflexes)were used in this trial, but measures were taken from both lower and upper limbs. The sum of 5 parameters of nerve conduction was used to evaluate large-fiber function of peripheral nerves. Norfolk QOL-DN and mBMI were also used. As the group of non-Val3OMet patients comprised of different phenotypical expressions, troponin I and $\mathrm{N}$-terminal prohormone of brain natriuretic peptide (NT-proBNP) cardiac biomarkers were used as outcomes. Several echocardiographic parameters [i.e., left-ventricular (LV) wall thickness, interventricular septal thickness, left-atrial diameter, LV mass, LV ejection fraction, and ratio of mitral peak velocity of early filling to early diastolic mitral annular velocity] and 12-lead EKG were also recorded [17].

Of 21 patients enrolled, 18 completed the trial [17]. The baseline demographics of patients demonstrated that this group of non-Val30Met patients had longer average disease duration (mean 64.7 months) and were of a more advanced age (mean 59.3 months) than the Val30Met patients enrolled in the previous studies. They demonstrated significant neurological and functional impairment in contrast with the early presentation of the disease in the previous populations. In addition, they represented a considerably heterogeneous population in which eight different TTR non-Val3OMet mutations were 
observed with mixed neurological and CM presentation [17].

TTR stabilization at week 6 was achieved in 18 $(94.7 \%)$ of 19 of the ITT population (two patients could not be evaluated) [17]. One patient who did not present TTR stabilization had a TTR Gly47Ala mutation; assessed again at months 3 and 6 , this patient then achieved stabilization. After 12 months of treatment, the mean increase in total NIS was 5.3 (NIS score ranges from 0 to 240). Patients with higher baseline NIS $(>70)$ presented a greater increase in NIS than patients with lower baseline scores. A small increase of 0.2 in nerve conduction summated score ( $\sum 5 \mathrm{NCs}$ nds) was observed after 12 months and the results were similar when excluding those who presented with maximal abnormality in nerve function since baseline [17].

Mean changes in Norfolk QOL-DN from baseline at months 6 and 12 were negligible [17]. The overall nutritional status was maintained over 12 months with variation between the 6-month and 12-month analyses. In patients with an interventricular septal wall $>12 \mathrm{~mm}$ at baseline, no clinically significant change was observed. Mean NT-proBNP values were elevated at baseline and remained stable at month 12. Troponin I was normal at baseline and remained stable throughout. Patients that were considered at a high risk of heart failure at baseline, defined as levels of NT-proBNP $>1000 \mathrm{pg} / \mathrm{ml}$, had a median increase in NT-proBNP levels of $521 \mathrm{pg} / \mathrm{ml}$ as compared to $47 \mathrm{pg} / \mathrm{ml}$ in patients with a low likelihood of heart failure at baseline. Adverse events were mostly related to the severity of the disease. Abnormalities in echocardiography and EKGs were considered consistent with cardiac morbidity [17]. Long-term data and controlled trials on evaluating more specifically cardiac function are expected.
Fx1A-303

Interim results were presented at the European Federation of Neurological Societies (EFNS) meeting in 2014 from the ongoing Fx1A-303 study [18]. Fx1A-303 is an open-label trial that enrolled patients who either received tafamidis in the single-treatment arm of the Fx1A-201 study (all non-Val30Met) [17], or completed the 18-month placebo-controlled Fx-005 trial [13] and then received tafamidis in the 12-month Fx-006 extension study (all Val30Met) [16].

Tafamidis was generally well tolerated [18]. All patient groups experienced some disease progression; those with Val30Met in the tafamidis-tafamidis arm had numerically smaller increases in NIS-LL and Norfolk QOLDN scores than those in the placebo-tafamidis arm at 66 months. Furthermore, once patients in the placebo-tafamidis arm began treatment with tafamidis, their apparent rate of increase in NISLL scores was similar to those in the tafamidistafamidis arm. Non-Val30Met patients had a higher disease burden at baseline and increases in NIS-LL scores at 48 months in a similar way to those seen in the Val30Met patients at 66 months. Long-term use of tafamidis in Val30Met patients was associated with less advancement in polyneuropathy impairment; however, the lack of a placebo control group hampers the interpretation of the efficacy results in non-Val30Met patients [18].

\section{POST HOC ANALYSES OF TAFAMIDIS}

\section{Impact of Tafamidis on Nutritional Status}

In 2014, Suhr et al. [19] published a post hoc analysis of a more detailed account of the impact of tafamidis on nutritional status of patients with TTR-FAP enrolled in clinical trials. 
mBMI was used as a physiological indicator for treated patients as it is closely related to the duration of gastrointestinal disturbances, malabsorption, and survival after LT [19].

For previous trials, mBMI was calculated as the product of the BMI and serum albumin concentration [19]. Height, weight, and albumin were measured during the baseline visit, and weight and serum albumin were measured every 6 months up to month 30 . The ITT population of 125 patients in the randomized phase of the trial was reduced to 71 patients in the extension study due to reasons previously described. Baseline evaluation of both groups of patients (tafamidis and placebo) showed that $70 \%$ presented gastrointestinal symptoms but that mean values of mBMI were normal as the patient groups constituted of only early-stage patients [19].

For the placebo-controlled phase of the trial, placebo recipients experienced a worsening in mBMI yielding a change from baseline to month 18 of $-33 \pm 16(P=0.04$; worsening of $1020 \pm 244 \mathrm{~kg} / \mathrm{m}^{2}$ by $\mathrm{g} / \mathrm{l}$ at each visit) [19]. In contrast, the recipients of tafamidis had a change of mBMI from baseline at 18 month of $+37 \pm 14 \quad(P=0.01 ; \quad$ improvement of $1032 \pm 167 \mathrm{~kg} / \mathrm{m}^{2}$ by $\mathrm{g} / \mathrm{l}$ at each visit), representing a LS mean difference of $73 \pm 21 \mathrm{~kg} / \mathrm{m}^{2}$ by $\mathrm{g} / \mathrm{l}$ between groups $(P=0.001)$. In conclusion of the open-label extension phase, placebo-treated patients had a change of mBMI from baseline of $+28 \pm 19 \mathrm{~kg} / \mathrm{m}^{2}$ by $\mathrm{g} / \mathrm{l}$ while tafamidis-treated patients had a change of $+16 \pm 18 \mathrm{~kg} / \mathrm{m}^{2}$ by g/l. The effect of the reduction in sample size during the extension phase influenced the ability to achieve statistical significance. These results were consistently comparable across study centers. The reversal of mean mBMI decrements observed for the placebo-treated patients during the open-label phase occurred because of improvements in both components of the index (BMI and albumin) [19].

A second analysis performed was the effect of initial BMI on changes in BMI from baseline [19]. Patients were divided into three groups: Underweight (BMI $<20 \mathrm{~kg} / \mathrm{m}^{2}$ ), normal (BMI $20-25 \mathrm{~kg} / \mathrm{m}^{2}$ ), and overweight (BMI $>25 \mathrm{~kg}$ / $\mathrm{m}^{2}$ ). Tafamidis-treated patients who were underweight gained in BMI at 18 months $\left(1.5 \pm 0.6 \mathrm{~kg} / \mathrm{m}^{2}\right), \quad$ normal-weight patients gained less $\left(0.5 \pm 0.3 \mathrm{~kg} / \mathrm{m}^{2}\right)$, and overweight patients had a decrease in BMI $(-0.5 \pm 0.4 \mathrm{~kg} /$ $\mathrm{m}^{2}$ ). For patients treated with the placebo, BMI remained constant in the three groups with a tendency for increased loss in overweight patients [19].

In the open-label extension phase, the tafamidis-tafamidis group at month 30 presented moderate increases in BMI for underweight $\quad\left(0.6 \pm 0.9 \mathrm{~kg} / \mathrm{m}^{2}\right) \quad$ and normal-weight $\quad\left(0.5 \pm 0.4 \mathrm{~kg} / \mathrm{m}^{2}\right)$ patients, while the overweight group showed moderate decreases $\left(-0.4 \pm 0.6 \mathrm{~kg} / \mathrm{m}^{2}\right)$ [19]. The patients in the placebo-tafamidis group who were underweight remained stable. The normal group showed a moderate increase in BMI $\left(0.8 \pm 0.6 \mathrm{~kg} / \mathrm{m}^{2}\right)$, whereas overweight patients showed a small decrease in BMI $(-0.1 \pm 0.5 \mathrm{~kg} /$ $\mathrm{m}^{2}$ ). The main finding was the improvement in or maintenance of nutritional status associated with tafamidis use. Interestingly, BMI increase associated with tafamidis was largely confined to those with relatively low values at baseline in whom improvement is desired as opposed to overweight patients [19].

\section{Tafamidis and Management of the Cardiac Aspects of TTR-FAP}

Another post hoc analysis addressed the QT interval of the EKG study [20] in healthy 
volunteers to assess the potential for QTc interval prolongation during tafamidis treatment at supra-therapeutic doses (400 mg single dose). The purpose of the study was the potential use of tafamidis in CM. The study demonstrated that administration of tafamidis $400 \mathrm{mg}$ in a single daily dose did not prolong QTc [20].

\section{Fx1B-201}

Results were recently published for the phase II, open-label Fx1B-201 study [21] in which TTR-CM patients received $20 \mathrm{mg}$ once daily of tafamidis. Tafamidis was used for 12 months together with the routine standard of care. Thirty-one WT-CM patients and 4 TTR V122I-CM patients were included. The mean age of patients was 76 years and their mean disease duration was 8 years. Ninety-four percent of patients had mild to moderate cardiac dysfunction (class I or II of NYHA classification). Kaplan-Meier analysis of survival from time of diagnosis was $87.5 \%$ survival rate at 30 months. Overall, 5.7\% subjects died, $25.7 \%$ experienced at least one cardiovascular-related hospitalization, and $25.7 \%$ experienced the composite endpoint of death or cardiovascular-related hospitalization. Cardiac biomarkers were elevated at baseline and increased over 12 months while troponin I and T remained relatively stable in the WT population. NYHA classification and 6-min walk test functional assessments supported the previous results. NYHA was improved or preserved for $75 \%$ of patients after 12 months and the overall population demonstrated minimal changes in the 6-min walk test (mean change from baseline of $-11 \mathrm{~m})$. Thirty of 31 patients $(96.8 \%)$ achieved TTR stabilization after 6 weeks, and 25/28 patients (89.3\%) after 12 months [21]. This is in contrast with a previous study on a prospective evaluation of the morbidity and mortality of WT and V122I-mutant TTR amyloid CM: The Transthyretin Amyloidosis Cardiac Study (TRACS; mean change from baseline in 6-min walk test of $-44 \mathrm{~m}$ ) [22].

The above studies in part provided the basis for the rationale to evaluate tafamidis for the treatment of TTR-CM. The ATTR-ACT trial [24] is an ongoing phase III, randomized study of tafamidis versus placebo for the treatment of TTR-CM [23].

\section{REAL-WORLD CLINICAL EXPERIENCE}

France was the first country to approve tafamidis for the treatment for patients with stage 1 TTR-FAP to slow disease progression in September 2011. According to Adams et al. [24, 25], tafamidis is an option for early-stage symptomatic TTR-FAP with positive amyloid biopsy. Treatment should be initiated and monitored by an experienced medical doctor. At each 6-month assessment, a complete multisystem approach is used and decisions are made as whether to pursue with tafamidis or to choose another option, including LT. Remaining on the waiting list for LT in the meanwhile is presented as an option. Decisions to change treatment are based on an increase in disease stage (1-3; Coutinho et al. [26]), an increase of one point in polyneuropathy disability (PND) score, an increase in one point for NYHA, conduction abnormalities on an EKG, or the presence of impotence and orthostatic hypotension [24, 25].

Lozeron et al. [27] studied the effect of tafamidis in predominantly late-onset Val30Met TTR-FAP patients in a prospective, non-randomized-controlled trial carried out at one of the French national reference centers. Thirty-seven patients were enrolled between December 2009 and July 2011 and assessed by 
NIS and disability scores. During the first 6 months of treatment, the mean progression of NIS-LL score was similar to that observed during the period before treatment. During the first year, $55 \%$ of patients deteriorated with respect to disability and $38 \%$ with respect to NIS. The authors concluded that, in most advanced Val30Met TTR-FAP, tafamidis was not able to stop disease progression with respect to NIS and disability [27].

In late-onset cases, a different composition of amyloid deposit has been shown in most tissues, especially in the heart, as opposed to early-onset cases. In work by Koike et al. [28], amyloid deposits were examined postmortem in eight cases. In early-onset cases, deposits were highly congophilic and had long, parallel fibrils in most organs. In late-onset cases, deposits were generally weakly congophilic and had short, haphazard fibrils, mostly like deposits from native TTR and similar to that observed in senile systemic amyloidosis (SSA), suggesting a different mechanism for organ damage. In addition, the amount of amyloid deposition in the peripheral nervous system was small for the severity of nerve fiber loss in late-onset cases, as was shown in another postmortem study from the same authors [29], precluding a mechanism of damage that goes beyond the deposit itself and should be taken into consideration if we are to put the effects of tafamidis into late-onset cases into the right context.

Authors, including Ueda and Ando [30] and Sekijima [31], have also considered tafamidis as a treatment option for patients with TTR-FAP since its approval by the Japanese Pharmaceuticals and Medical Devices Agency in 2013 for TTR-FAP (any stage). Nevertheless, Ueda and Ando [30] highlight the need to follow long-term outcomes, cardiac functions, and ophthalmic symptoms in patients treated with tafamidis.
Obici and Merlini [32], in their review of treatment options for TTR-FAP, indicate tafamidis as a treatment option for patients with stage 1 TTR-FAP of Coutinho [26], PND $\leq$ II. In those patients, liver or combined liver-heart transplantation is often approached as a second-line option when disease progression has occurred while on tafamidis treatment. Consistently, participation into a placebo-controlled trial is often considered only after inadequate response to tafamidis.

Barbosa da Silva et al. [33] compared the disease progression of early-stage Val3OMet TTR-FAP patients treated with tafamidis or submitted to LT with that of untreated patients from two referral centers-CEPARM, Rio de Janeiro, Brazil and Henri-Mondor, Paris, France-included in the Transthyretin Amyloidosis Outcome Survey (THAOS) database. The main inclusion criteria were: the presence of Val3OMet TTR mutation; a biopsy positive for amyloid deposit; a PND score of I or II; treatment or nontreatment after 2007 (the date inclusion into the THAOS database was commenced); and at least two independent medical evaluations within a minimum of 1 year (maximum 4 years). The assessment methods used were NIS (any increase $>2$ points was considered deterioration), and Karnofsky and PND scores (any increase was considered deterioration). Twenty-two patients were selected, of whom 13 were Brazilian and 9 were French, and were divided into three distinct groups: 8 patients submitted to LT, 7 using tafamidis, and 7 who were not treated. Of them 77.27\% were in PND stage I and 22.73\% were in stage II. The median evaluation period of patients submitted to LT was 3 (range 1-5) years, treated with tafamidis was 3 (range 2-5) years, and with no treatment was 2.5 (range 1-4) years. The three groups had similar demographic and clinical profiles, based on age, 
disease severity, the duration of disease, and the period of treatment. Gender was distributed equally between the groups [33].

NIS scores showed no deterioration in $42.9 \%$ of the tafamidis-treated group, as opposed to $25 \%$ in LT group and $14.9 \%$ in the no-treatment group [33]. Karnofsky score showed no deterioration in $42.86 \%$ of patients treated with tafamidis, $75 \%$ of the LT group, and $14.29 \%$ of the no-treatment group. According to PND scores, patients treated with tafamidis and LT indicated no deterioration in PND, $85.72 \%$ and $87.5 \%$, respectively, against $28.57 \%$ of the no-treatment group. Considering the restraints of the study (with a small population and retrospective analysis), it was the first to compare LT to tafamidis treatment between comparable groups of patients. After a follow-up of 2-6 years in this small population study, similar neurological outcomes were observed in the tafamidistreated and LT groups when compared to the untreated group, using the Karnofsky and PND scores as an evaluation method. Both had a better clinical course in relation to the natural evolution of the disease [33]. Controlled prospective studies comparing both treatments would be very useful but also very difficult to achieve.

Coelho et al. [34], from the two most important reference centers in Portugal (Porto and Lisboa), presented their 1-year efficacy and safety experience of treatment with tafamidis. Overall, 122 patients were evaluated at baseline and then every 6 months through a comprehensive protocol that included NIS score, BMI, Norfolk QOL-DN score, and renal, thyroid and liver function tests. Adverse events were also recorded. Patients were classified as responders if NIS changes across 12 months were $<2$, according to Dyck's classification. BMI, liver, renal and thyroid function tests remained stable after 1-year of follow-up. Norfolk QOL-DN score improved from baseline to 1 year (3.21 vs. 2.89, respectively, $P<0.01)$. Mean NIS changed from baseline to 6 months (2.45 vs. 2.51, respectively, $P<0.01)$ and stabilized between 6 and 12 months (2.51 vs. 2.54 , respectively, $P<0.01)$, showing a delay in the stabilization effect as occurred in the main trials. Patients that were classified as responders $(n=75.61 \%)$ showed a significant decrease in NIS score between 6 and 12 months (2.41 vs. 2.33, respectively, $P<0.01$ ) [32]. Tafamidis prevented neurological, BMI, and QOL deterioration in $61 \%$ of the cases treated for one year.

\section{FUTURE DIRECTIONS}

Experts and patients are currently awaiting the long-term results obtained so far from treatment with tafamidis, as well as it safety profile, so that these can be comparable to the results of LT, of which we have a long experience of more than 20 years to demonstrate its efficacy [3, 35]. Also to be noted is that the mortality and morbidity associated with LT and subsequent immunosuppression, as well as the availability of organs and the well-documented continuous deposition of amyloid in tissues (mainly in the heart) coming from native TTR after LT, support the need for an alternative option to LT.

The recent publication from Maia et al. [36] indicates that central nervous system (CNS) clinical involvement occurs in Val30Met TTR-FAP patients regardless of LT. Long-term CNS involvement was monitored in 87 consecutive Val30Met TTR-FAP and 35 non-TTR-FAP LT patients investigated with computed tomography scan, electroencephalography, and extensive neurovascular workup. Focal neurological episodes occurred in 31\% (27/87) of Val30Met TTR-FAP patients and in $5.7 \%(2 / 35)$ of the 
non-TTR-FAP LT patients. CNS TTR-FAP progressed from the meninges and its vessels toward meningocortical vessels and the superficial brain parenchyma, as disease duration increased. Longer disease duration after LT can provide the necessary time for TTR-FAP to progress [36]. Another limitation of LT is that if the age of a patient is greater than 65 years then this usually constitutes an exclusion criterium in most centers, thus making tafamidis a good safe alternative for late-onset cases [37].

The impact of tafamidis on cardiac disease is also of great interest as little is known about its long-term effect. Cardiac data previously arose from the Fx1A-201 trial [17], although recently published data from the Fx1B-201 study [21], is encouraging further evaluation of tafamidis for TTR-CM in an ongoing worldwide phase III double-blind, randomized, placebo-controlled study of tafamidis in up to 400 patients with TTR-CM (NCT01994889) [23]. The efficacy of tafamidis for SSA will be partially determined after the conclusion of this trial. As tafamidis is a safe drug and equally capable of stabilizing mutant and native TTR, interest is increasing for this potential treatment for a senile population. In addition, there is no knowledge regarding the impact of tafamidis on renal disease, which would have a very important, positive effect on long-term survival of patients with ATTR.

According to the results of trials and real-world experience $[13,16-21,23-25,27$, 30-35], the use of tafamidis in very early symptomatic/asymptomatic TTR-FAP patients, before the overt onset of the disease, would benefit most from its effect on disease progression-the most important goal at the present moment. To achieve this, medical doctors and patient communities are fostering searches for early disease markers, carriers of the mutation in families, and final diagnosis of suspected and non-suspected cases (late-onset and sporadic cases for instance).

To foster the increase in the ability to recognize new patients and carriers, it is crucial to spread the knowledge of the disease and make diagnostic tools available. In developed countries, local networks of medical doctors work well. Referral centers act by centralizing data and preceptorship. In underdeveloped countries, the challenge is far more difficult as teaching about a rare disease faces poor health systems and the complete lack of diagnostic means, in an unfavorable social and economic environment. In past years, countries like those in Latin America are slowly recognizing the presence of TTR-FAP with various presentations and multiple genetic backgrounds and the interest in this extremely underdiagnosed disease is definitely increasing.

Late-onset and sporadic cases are very challenging to diagnose but the availability of new tools for diagnosis and the increase in the awareness of this disease are enhancing our capability of detecting those cases very frequently misdiagnosed as idiopathic or chronic inflammatory demyelinating polyneuropathy, to mention just a few. Their recognition will enable a much earlier initiation of therapy than is the usual case and hence improve the prognosis after treatment [38].

Another question of interest is the effect of tafamidis on preventing the development of amyloidosis in the recipient of a domino LT, as well as its use as a combined treatment with LT. There is awareness of some patients being treated in these contexts but so far no results have been reported.

A randomized clinical trial was reported in 2013 where diflunisal was shown to reduce the rate of progression of neurologic impairment and to preserve patient QOL at the 2-year follow-up period of the study [39]. This study 
included both Val30Met TTR-FAP and non-Val30Met TTR-FAP patients and therefore cases with phenotypical variety including both early and late onset. The safety profile was considered satisfactory. Several patients are using diflunisal in real-world practice although it is not yet available in several parts of the world thus limiting the gain of experience. A long-term report looking at both the maintenance of the beneficial effect of diflunisal and mostly its satisfactory safety profile is still awaited, especially as it is an anti-inflammatory drug that would be used for a long period or for a lifetime in a group of patients experiencing $\mathrm{CM}$ and renal disfunction with relatively high frequency [39]. This will help the physician in care of the patient to decide which TTR stabilizer to use.

At least two important and promising clinical trials with agents capable of reducing TTR production, either by RNAi-targeting messenger, RNA transcribed from TTR gene [40], or by anti-sense oligonucleotide drugs designed to target human TTR mRNA, are enrolling FAP/FAP-CM patients. The capacity of both novel drugs to suppress TTR protein levels with a mean reduction of around $75 \%$ at the chosen doses determined in previous phase I/II trials [40] is unquestionable. To determine their efficacy composite endpoints including neurological, neurophysiological, nutritional, autonomic, and cardiac aspects will be used. In the future, patients and experts will certainly have different treatment options, either alone or in combination, depending on the presentation or stage of the disease.

\section{CONCLUSION}

Tafamidis has opened the way for a nonsurgical treatment possibility for TTR-FAP, being both safe and efficacious in treating early-stage patients, and a practical oral drug to be prescribed for a lifetime. In the future, tafamidis will potentially find a more important use as a drug capable of delaying or arresting TTR-FAP expression as much as possible; however, this will be dependent on its proper use at the correct moment, that is, especially before the overt expression of TTR-FAP. The last task will be the more challenging to achieve unless a robust biomarker for TTR-FAP onset is identified.

\section{ACKNOWLEDGMENTS}

No funding or sponsorship was received for this study or publication of this article. All named authors meet the International Committee of Medical Journal Editors (ICMJE) criteria for authorship for this manuscript, take responsibility for the integrity of the work as a whole, and have given final approval for the version to be published. During the peer review process, the manufacturer of the agent under review was offered an opportunity to comment on the article. Changes resulting from comments received were made by the author based on their scientific and editorial merit.

\section{Compliance with Ethical Standard}

Conflict of interest Márcia Waddington Cruz served as an advisor for Pfizer Inc., as well as having served as an advisor and received support as a clinical investigator from FoldRx Pharmaceuticals, which was acquired by Pfizer Inc. in October 2010. She currently serves on the THAOS (a disease registry capturing natural history and effect of the therapies on TTR amyloidosis) scientific advisory board. Merril D. Benson has served as a consultant from Pfizer Inc. for teaching programs and design of research. 
Compliance with ethics guidelines This article is based on previously conducted studies and does not involve any new studies of human or animal subjects performed by any of the authors.

Open Access This article is distributed under the terms of the Creative Commons Attribution-NonCommercial 4.0 International License (http://creativecommons.org/licenses/ by-nc/4.0/), which permits any noncommercial use, distribution, and reproduction in any medium, provided you give appropriate credit to the original author(s) and the source, provide a link to the Creative Commons license, and indicate if changes were made.

\section{REFERENCES}

1. Cruz MW. Regional differences and similarities of familial amyloidotic polyneuropathy (FAP) presentation in Brazil. Amyloid. 2012;19(Suppl. 1):65-7.

2. Benson MD, Kincaid JC. The molecular biology and clinical features of amyloid neuropathy. Muscle Nerve. 2007;36:411-23.

3. Ericzon BG, Wilczek HE, Larsson M, et al. Liver transplantation for hereditary transthyretin amyloidosis: after 20 years still the best therapeutic alternative? Transplantation. 2015;0:1-8.

4. Johnson ST, Connelly S, Fearns C, Powers ET, Kelly JW. The transthyretin amyloidoses: from delineating the molecular mechanism of aggregation linked to pathology to a regulatory agency approved drug. J Mol Biol. 2012;421:185-203.

5. Bulawa CE, Connelly S, DeVit M, et al. Tafamidis, a potent and selective transthyretin kinetic stabilizer that inhibits the amyloid cascade. PNAS. 2012;24:9629-34.

6. Berk J, Dyck P, Obici L, et al. The diflunisal trial: update on study drug tolerance and disease progression. Amyloid. 2011;18(Suppl. 1):191-2.

7. Hammarstrom P, Jiang X, Hurshman AR, Powers ET, Kelly JW. Sequence-dependent denaturation energetics: a major determinant in amyloid disease diversity. PNAS. 2002;99:16427-32.
8. Kelly JW, Colon W, Lai Z, et al. Transthyretin quaternary and tertiary structural changes facilitate misassembly into amyloid. Adv Protein Chem. 1997;50:161-81.

9. McCutchen SL, Lai Z, Miroy GJ, Kelly JW, Colon W. Comparison of lethal and nonlethal transthyretin variants and their relationship to amyloid disease. Biochemistry. 1995;34:13527-36.

10. McCutchen SL, Colon W, Kelly JW. Transthyretin mutation Leu-55-Pro significantly alters tetramer stability and increases amyloidogenicity. Biochemistry. 1993;32:12119-27.

11. Coelho T, Carvalho M, Saraiva MJ, Alves I, Almeida MR, Costa PP. A strikingly benign evolution of FAP in an individual found to be a compound heterozygote for two TTR mutations: TTR MET 30 and TTR MET 119. J Rheumatol. 1993;20:179.

12. Coelho T, Chorao R, Sousa A, Alves I, Torres MF, Saraiva MJ. Compound heterozygotes of transthyretin Met30 and transthyretin Met119 are protected from the devastating effects of familial amyloid polyneuropathy. Neuromusc Disord. 1996;6:27.

13. Coelho T, Maia LF, da Silva AM, et al. Tafamidis for transthyretin familial amyloid polyneuropathy. A randomized, controlled trial. Neurology. 2012;79:785-92.

14. Dyck PJ, Karnes JL, O’Brien PC, Litchy WJ, Low PA, Melton LJ 3rd. The rochester diabetic neuropathy study: reassessment of tests and criteria for diagnosis and staged severity. Neurology. 1992;42:1164-70.

15. Dyck PJ, Davies JL, Litchy WJ, O'Brien PC. Longitudinal assessment of diabetic polyneuropathy using a composite score in the rochester diabetic neuropathy study cohort. Neurology. 1997;49:229-39.

16. Coelho T, Maia LF, da Silva AM, et al. Long-term effects of tafamidis for the treatment of transthyretin familial amyloid polyneuropathy. J Neurol. 2013;260:2802-14.

17. Merilini G, Planté-Bordeneuve V, Judge DP, et al. Effects of tafamidis on transthyretin stabilization and clinical outcomes in patients with Non-Val30et transthyretin amyloidosis. J Cardiovasc Trans Res. 2013;6:1011-20.

18. Coelho T, Conceição IM, Barroso F, et al. Long term effects of tafamidis treatment on transthyretin familial amyloid polyneuropathy (TTR-FAP): interim results from the Fx1A-303 study. Eur J Neurol. 2014;21(Suppl. 1):81. 
19. Suhr OB, Conceição IM, Karayal ON, Mandel FS, Huertas PE, Ericzon BG. Post Hoc analysis of nutritional status in patients with transthyretin familial amyloid polyneuropathy: impact of tafamidis. Neurol Ther. 2014;3:101-12.

20. Klamerus KJ, Watsky E, Moller R, Wand R, Riley S. The effect of tafamidis on the QTc interval in heathy subjects. $\mathrm{Br} \mathrm{J}$ Clin Pharmacol. 2015;79(6):918-25. doi:10.1111/bcp.12561.

21. Maurer MS, Grogan DR, Judge DP, Mundayat R, Packman J, Lombardo I, Quyyumi AA, Aarts J, Falk RH. Tafamidis in transthyretin amyloid cardiomyopathy: effects on transthyretin stabilization and clinical outcomes. Circ Heart Fail. 2015; CIRCHEARTFAILURE.113.000890published online before print April 142015.

22. Ruberg FL, Maurer MS, Judge DP, et al. Prospectie evaluation of the morbidity and mortality of wild-type and V122I mutant transthyretin amyloid cardiomyopathy: the transthyretin amyloidosis cardiac study (TRACS). Am Heart J. 2012;164:222-8.

23. Safety and pharmacokinetic assessment of orally administered tafamidis in healthy volunteers. Available from: https://clinicaltrials.gov/ct2/show/ NCT01994889?term $=\% 22$ tafamidis\%22+and+\%22 cardiomyopathy $\% 22 \&$ rank $=1$. Safety and efficacy of tafamidis in patients with transthyretin cardiomyopathy (ATTR-ACT).

24. Adams D. Recent Advances in the treatment of familial amyloid polyneuropathy. Adv Neurol Disord. 2013;6:139-49.

25. Adams D, Cauquil C, Theaudin M, Rousseaus A, Algalarrondo V, Slama MS. Current and future treatment of amyloid neuropathies. Expert Rev Neurother. 2014;14:1437-51.

26. Coutinho P, Martins da Silva A, Lopes Lima J, Resende Barbosa A. Forty years of experience with type I amyloid neuropathy. Review of 483 cases. In: Glenner GG, Pinho P, Costa E, Falcao de Freitas A (Eds.), Amyloid and amyloidosis. Amsterdam: Excerpta Medica; 1980. pp. 88-98.

27. Lozeron $\mathrm{P}$, Théaudin $\mathrm{M}$, Mincheva $\mathrm{Z}$, Duccot $\mathrm{B}$, Lacrox C, Adams D, French Network for FAP (CORNAMYL). Effect on disability and safety of Tafamidis in late onset of Met30 transthyretin familial amyloid polyneuropathy. Eur J Neurol. 2013;20:1539-45.

28. Koike H, Ando Y, Ueda M, Kawagashira Y, Iijima M, Fujitake J, Hayashi M, Yamamoto M, Mukai E, Nakamura T, Katsuno M, Hattori N, Sobue G. Distinct characteristics of amyloid deposits in early- and late-onset transthyretin Val30 Met familial amyloid polyneuropathy. J Neurol Sci. 2009;287:178-84.

29. Koike H, Misu K, Sugiura M, Iijima M, Mori K, Yamamoto M, Hattori N, Mukai E, Ando Y, Ikeda S, Sobue G. Pathology of early- versus late-onset TTR Met30 familial amyloid polyneuropathy. Neurology. 2004;63(1):129-38.

30. Ueda M, Ando Y. Recent advances in transthyretin amyloidosis therapy. Transl Neurodegener. 2014;3:19.

31. Seikijima Y. Recent progress in the understanding and treatment of transthyretin amyloidosis. J Clin Pharm Ther. 2014;39:225-33.

32. Obici L, Merlini G. An overview of drugs current under investigation for the treatment of transthyretin-related hereditary amyloidosis. Expert Opin Investig Drugs. 2014;23:1239-51.

33. Barbosa da Silva RV, Cruz MW, Planté-Bordeneuve V. Follow-up of transthyretin amyloidosis patients with liver transplants or receiving tafamidis treatment as documented in THAOS: the transthyretin amyloidosis outcomes survey. Poster presented at the 16th Congress of the European Federation of Neurological Societies. Stockholm, Sweden, 8-11, 2012. P 2918.

34. Coelho T, Conceição I, Cardoso M, et al. Evaluation of 1 year treatment with tafamidis in Portuguese patients with transthyretin familial amyloid polyneuropathy. Eur J Neurol. 2014;21(Suppl. 1):327.

35. Ando Y, Coelho T, Berk JL, et al. Guideline of transthyretin-related hereditary amyloidosis for clinicians. Orphanet J Rare Dis. 2013;8:31.

36. Maia LF, Magalhães R, Freitas J, Taipa R, Pires MM, Osório H, Dias D, Pessegueiro H, Correia M, Coelho $\mathrm{T}$. CNS involvement in $\mathrm{V} 30 \mathrm{M}$ transthyretin amyloidosis: clinical, neuropathological and biochemical findings. J Neurol Neurosurg Psychiatry. 2015;86(2):159-67. doi:10.1136/jnnp2014-308107 (Epub 2014 Aug 4).

37. Okamoto S, Wixner J, Obayashi K, Ando Y, Ericzon BG, Friman S, Uchino $M$, Suhr OB. Liver transplantation for familial amyloidotic polyneuropathy: impact on Swedish patients' survival. Liver Transpl. 2009;15:1229-35.

38. Koike H, Hashimoto R, Tomita M, Kawagashira Y, Iijima M, Tanaka F, Sobue G. Diagnosis of sporadic transthyretin Val30Met familial amyloid polyneuropathy: a practical analysis. Amyloid. 2011;18:53-62.

39. Berk JL, Suhr OB, Obici L, et al. Repurposing diflunisal for familial amyloid polyneuropathy: a randomized clinical trial. Jama. 
2013;310(24):2658-2667. Prepublished on 2013/12/26 as doi:10.1001/jama.2013.283815. PMID24368466.

40. Coelho T, Adams D, Silva A, et al. Safety and efficacy of RNAi therapy for transthyretin amyloidosis. N Engl J Med. 2013;369:819-29.
41. Hammarstrom $P$, Jiang $X$, Hurshman AR, Powers ET, Kelly JW. Sequence-dependent denaturation energetics: A major determinant in amyloid disease diversity. PNAS. 2002;99:16427-32. 